\title{
Research on Time-delay Vibration Reduction Control of 1/4 Vehicle Model
}

\section{Yongguo Zhang*, Kehui Ma, Zhen Xu, Yajie Chen and Pengcheng Zhou}

\author{
School of Transportation and Vehicle Engineering \\ Shandong University of Technology, Zibo 25500, China
}

*Corresponding author details: Yongguo Zhang; 17853465307@163.com

\begin{abstract}
In this paper, a $1 / 4$ vehicle suspension model with time-delay state feedback control is established, and the maximum time-delay allowed by different feedback gains in the control system is solved according to the Lyapunov stability theory. The stability of the vehicle suspension system is analyzed, and the vibration characteristics of the system are analyzed in the frequency domain and time domain. The simulation results show that the time-delay feedback control can effectively improve the damping performance of the vehicle suspension and improve the ride comfort of vehicle.
\end{abstract}

Keywords: time-delay feedback; active suspension; time-delay stability; simulation

\section{INTRODUCTION}

In the process of driving, the vibration caused by the road roughness is transmitted to the goods and passengers through the suspension, which affects the integrity of the goods and comfort of the passengers. Therefore, how to reduce body vibration has become a hot topic for experts at home and abroad. Time delay exists in various controllable active suspensions, and the small-time delay may cause system instability. However, time delay itself has inherent damping characteristics, and time delay can be used as an active control item to reduce vehicle vibration [1].

Olgac introduced time-delay displacement feedback into the traditional dynamic vibration absorber for the first time as partial state feedback to reduce the vibration of the main system, so it is called delayed resonator [2-3]. Jalili improved on the time-delay dynamic vibration absorber and proposed a time-delay feedback shock absorber for a specific frequency range, which was implemented through a nested identification and control optimization program [4]. The research results of Zhao et al. [5] show that the time-delay nonlinear dynamic vibration absorber has a wide range of stability control and good vibration reduction effect. However, for the optimization selection of parameters, the above literature only uses the trial method to verify and select. $\mathrm{Li}$ and Sun took the vehicle model with dynamic vibration absorber as the research object, and discussed the vibration reduction effects of $\mathrm{LQR}$ control and time-delay feedback control on the vehicle model. Through comparison and simulation, they found that the two methods have a better vibration control effect. [6] With the development of time-delay dynamics, proper introduction of time-delay in the control system of automobile suspension can reduce the vibration of the car and improve the stability of the car. Therefore, the time-delay has a great influence on the performance of the vehicle system [7].

In this paper, a two-degree-of-freedom time-delay damping suspension model is established on basis of $1 / 4$ vehicle model.
The tire time-delay state variable is used as the active suspension control parameter, and the influence of the timedelay control parameter on the suspension performance is analyzed. And verify the effect of the active suspension with time-delay feedback control in improving the ride comfort of the vehicle under the simple harmonic excitation of the road.

\section{MECHANICAL MODEL}

Simplify the vehicle according to the research purpose and establish a $1 / 4$ vehicle model, as shown in FIGURE $1 . m_{e}$ is the mass of the body; $k_{1}$ and $c_{1}$ represent the stiffness and damping coefficients of the suspension, respectively; $m_{c}$ is the mass of the tire; $k_{2}$ and $c_{2}$ are the stiffness and damping coefficient of the tire; $x_{d}$ is the road excitation; $x_{1}$ and $x_{2}$ represent the vertical displacement of the body and tires, respectively; $u$ is the time-delay feedback control force.

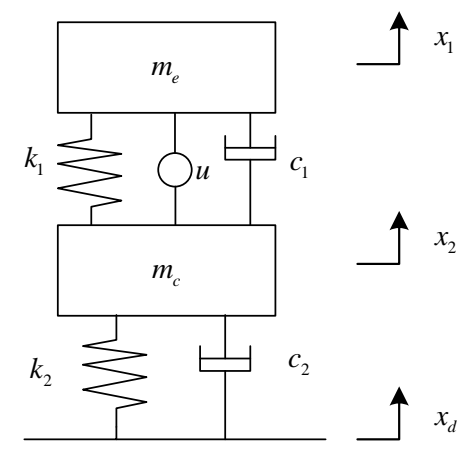

FIGURE 1: Model of 1/4 Vehicle Suspension System with Time-delay Feedback Control.

The dynamic equations of the system model are

$$
\begin{gathered}
m_{e} x^{\&^{\varepsilon_{1}}}+k_{1}\left(x_{1}-x_{2}\right)+c_{1}\left(x^{\&_{1}}-x^{\&_{2}}\right)+u=0 \\
m_{c} x^{\&^{\&_{2}}}+k_{2}\left(x_{2}-x_{d}\right)+c_{2}\left(x^{\&_{2}}-x^{\&_{d}}\right)-k_{1}\left(x_{1}-x_{2}\right)- \\
c_{1}\left(x^{\&_{1}}-x^{\&_{2}}\right)-u=0
\end{gathered}
$$


Where:

$u=g x_{2}(t-\tau), g$ is the feedback gain coefficient, $\tau$ is the time delay.

\section{ESTABLISHMENT OF SUSPENSION CONTROL SYSTEM}

\section{MODEL}

According to the performance requirements of vehicle suspension system, state variable

$X=\left[x_{1}, x^{\&_{1}}, x_{2}, x^{\&_{2}}\right]^{T}$ and output variable

$Y(t)=\left[x_{1}, x^{\varepsilon^{\varepsilon_{1}}}, x_{1}-x_{2}, x_{2}-x_{d}\right]^{T}$ are selected.

Then the state equation of the system is

$$
\left\{\begin{array}{l}
\dot{x} \&(t)=A X(t)+B U(t)+E w(t) \\
Y(t)=C X(t)+D U(t)+F w(t)
\end{array}\right.
$$

$$
\begin{aligned}
& \text { Where: } \\
& A=\left[\begin{array}{cccc}
0 & 1 & 0 & 0 \\
-\frac{k_{1}}{m_{e}} & -\frac{c_{1}}{m_{e}} & \frac{k_{1}}{m_{1}} & \frac{c_{1}}{m_{1}} \\
0 & 0 & 0 & 1 \\
\frac{k_{1}}{m_{c}} & \frac{c_{1}}{m_{c}} & -\frac{k_{1}+k_{2}}{m_{c}} & -\frac{c_{1}+c_{2}}{m_{c}}
\end{array}\right], B=\left[\begin{array}{c}
0 \\
-\frac{g}{m_{e}} \\
0 \\
\frac{g}{m_{c}}
\end{array}\right], \\
& C=\left[\begin{array}{cccc}
1 & 0 & 0 & 0 \\
-\frac{k_{1}}{m_{e}} & -\frac{c_{1}}{m_{e}} & \frac{k_{1}}{m_{e}} & \frac{c_{1}}{m_{e}} \\
1 & 0 & -1 & 0 \\
0 & 0 & 1 & 0
\end{array}\right], E=\left[\begin{array}{cc}
0 & 0 \\
0 & 0 \\
0 & 0 \\
\frac{k_{2}}{m_{c}} & \frac{c_{2}}{m_{c}}
\end{array}\right], \\
& D=\left[\begin{array}{c}
0 \\
-\frac{g}{m_{c}} \\
0 \\
0
\end{array}\right], F=\left[\begin{array}{cc}
0 & 0 \\
0 & 0 \\
0 & 0 \\
-1 & 0
\end{array}\right], w(t)=\left[\begin{array}{c}
x_{d} \\
x_{d}^{\&}
\end{array}\right], \\
& U(t)=x_{2}(t-\tau) .
\end{aligned}
$$

\section{SYSTEM STABILITY CONDITIONS}

When the time-delay feedback control is introduced into the system, only the appropriate feedback gain coefficient can be selected to ensure the stability of the system. Therefore, it is necessary to obtain the time-delay stability interval of the system.

Laplace transform is applied to equation (2), and the characteristic equation of the system is obtained as

$$
\operatorname{det}\left(\left[\begin{array}{ll}
a_{11} & a_{12} \\
a_{21} & a_{22}
\end{array}\right]\right)=0
$$

Where:

$$
\begin{aligned}
& a_{11}=m_{e} s^{2}+c_{1} s+k_{1}, a_{12}=-c_{1} s-k_{1}-g e^{-s \tau}, a_{21}=-c_{1} s-k_{1}, \\
& a_{22}=m_{c} s^{2}+k_{2}+c_{2} s+k_{1}+c_{1} s-g e^{-s \tau} .
\end{aligned}
$$

At present, the methods for analyzing the stability of timedelay systems mainly include frequency-domain methods and time-domain methods. This paper chooses the conventional Lyapunov stability theory, and judges the stability of the system through the pure imaginary roots of the characteristic equation of the system. If all the roots of the characteristic equation have negative real parts, the system is asymptotically stable. The critical condition for system instability is that the characteristic equation has pure imaginary roots $s=i \omega$.

Substitute $s=i \omega$ into equation (3), and use Euler's formula $e^{-i \omega \tau}=\cos (\omega \tau)-i \sin (\omega \tau)$ for substitution.

$$
\left\{\begin{array}{l}
\cos (\omega \tau)=\frac{k_{1} m_{c} \omega^{2}-k_{1} k_{2}+k_{1} m_{e} \omega^{2}+k_{2} m_{e} \omega^{2}-m_{c} m_{e} \omega^{4}+c_{1} c_{2} \omega^{2}}{g m_{e} \omega^{2}} \\
\sin (\omega \tau)=-\frac{c_{1} m_{c} \omega^{3}+c_{1} m_{c} \omega^{3}+c_{2} m_{e} \omega^{3}-c_{1} k_{2} \omega-k_{1} c_{2} \omega}{g m_{e} \omega^{2}}
\end{array}\right.
$$

According to the trigonometric relation $\cos ^{2}(\omega \tau)+$ $\sin ^{2}(\omega \tau)=1$, the equation for $\omega$ can be obtained

$$
a_{4} \omega^{8}+a_{3} \omega^{6}+a_{2} \omega^{4}+a_{1} \omega^{2}+a_{0}=0
$$

Where:

$$
\begin{aligned}
& a_{4}=m_{c}^{2}+m_{e}^{2}, a_{3}=c_{1}^{2} m_{c}^{2}+c_{1}^{2} m_{e}^{2}+c_{2}^{2} m_{e}^{2}-2 k_{1} m_{c} m_{e}^{2}-2 k_{1} m_{e} m_{c}^{2}- \\
& 2 k_{2} m_{c} m_{e}^{2}+2 c_{2} c_{1} m_{e}^{2}+2 m_{c} m_{e} c_{1}^{2}, a_{2}=c_{1}^{2} c_{2}^{2}-2 c_{1}^{2} k_{2} m_{c}-2 c_{1}^{2} k_{2} m_{e}- \\
& 2 c_{2}^{2} k_{1} m_{e}-g^{2} m_{e}^{2}+k_{1}^{2} m_{c}^{2}+2 k_{1}^{2} m_{e} m_{c}+k_{1}^{2} m_{e}^{2}+4 k_{1} k_{2} m_{e} m_{c}+ \\
& 2 k_{1} k_{2} m_{e}^{2}+m_{e}^{2} k_{2}^{2}, a_{1}=c_{1}^{2} k_{2}^{2}+c_{2}^{2} k_{1}^{2}-2 k_{1}^{2} k_{2} m_{c}-2 k_{1} k_{2}^{2} m_{c}- \\
& 2 k_{1} k_{2}^{2} m_{e}-2 k_{1}^{2} k_{2} m_{e}, a_{0}=k_{1}^{2} k_{2}^{2}
\end{aligned}
$$

The above equation defines the relationship equation $g=G(\omega)$ between $g$ and $\omega$, and substituting it into equation (5) can get the relationship between $\tau$ and $\omega$.

According to the parametric equation $\left\{\begin{array}{l}g=G(\omega) \\ \tau=T(\omega)\end{array}\right.$,

the critical time delay corresponding to the feedback gain coefficient within the value range of $w$ can be obtained, as shown in FIGURE 2.

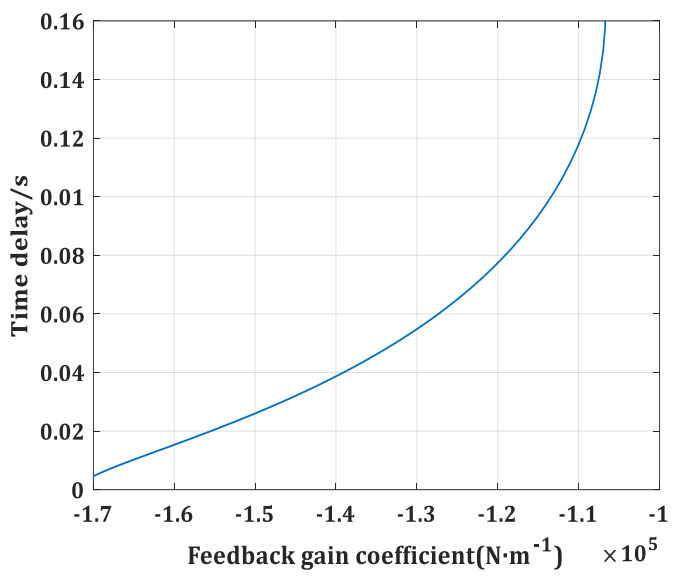

FIGURE 2: Critical Time Delay under Different Feedback Gain

The curve represents the critical time delay for system stability. Below the curve, the time-delay system is asymptotically stable. Above the curve, the time-delay system is unstable. When equation (5) has no real root, the system is stable for any time delay.

\section{SOLVE TIME-DELAY CONTROL PARAMETERS}

Fourier transformation of the dynamic equation can be obtained

$$
\left\{\begin{array}{l}
A_{11} X_{e}(\omega)+A_{12} X_{c}(\omega)=0 \\
A_{21} X_{e}(\omega)+A_{22} X_{c}(\omega)=\left(k_{2}+c_{2} i \omega\right) X_{d}(\omega)
\end{array}\right.
$$

Where:

$$
\begin{aligned}
& A_{11}=-m_{e} \omega^{2}+c_{1} i \omega+k_{1}, A_{12}=-c_{1} i \omega-k_{1}-g e^{-i \omega \tau}, \\
& A_{21}=-c_{1} i \omega-k_{1}, A_{22}=-m_{c} \omega^{2}+k_{2}+c_{2} i \omega+c_{1} i \omega+k_{1}-g e^{-i \omega \tau} .
\end{aligned}
$$

The amplitude-frequency characteristics of the car body are calculated as

$$
|H(\omega)|=\left|\frac{X_{c}(\omega)}{X_{b}(\omega)}\right|=\left|\frac{A_{12}\left(k_{2}+c_{2} i w\right)}{A_{12} A_{21}-A_{11} A_{22}}\right|
$$


Equation (7) is only a function of the feedback gain coefficient and time delay. According to the theory of differential equations, when the partial derivatives of equation (7) to $g$ and $\tau$ are zero, the function takes the extreme value [8].

$$
\frac{\partial|H(\omega)|}{\partial g}=\frac{\partial|H(\omega)|}{\partial \tau}=0
$$

Since equation (8) contains a transcendental function term, which does not satisfy any function of a polynomial equation with a polynomial as a coefficient, the equation will have infinitely many solutions. The corresponding $|H(\omega)|$ has an infinite number of extreme points.

According to the stability constraints, select the minimum points in the time-delay independent stability zone, and the corresponding ones are the optimal feedback parameters to be sought. And the minimum point close to the critical line of the stable region has a better damping effect.

Draw the curves of $\frac{\partial|H(\omega)|}{\partial g}=0$ and $\frac{\partial|H(\omega)|}{\partial \tau}=0$

respectively, as shown in FIGURE 3. The parameters of the vehicle model are shown in TABLE 1.

In the FIGURE (3), the solid line represents the extreme point distribution of the time delay value, while the dotted line represents the extreme point distribution of the feedback gain coefficient. The intersection point of the two curves is the solution of the system, and there are infinitely many periodic distribution solutions to the equation Combined with the stability conditions, the optimal feedback gain coefficient and time delay value can be obtained

$$
g=-22670 N \cdot m^{-1}, \tau=0.2419 s
$$

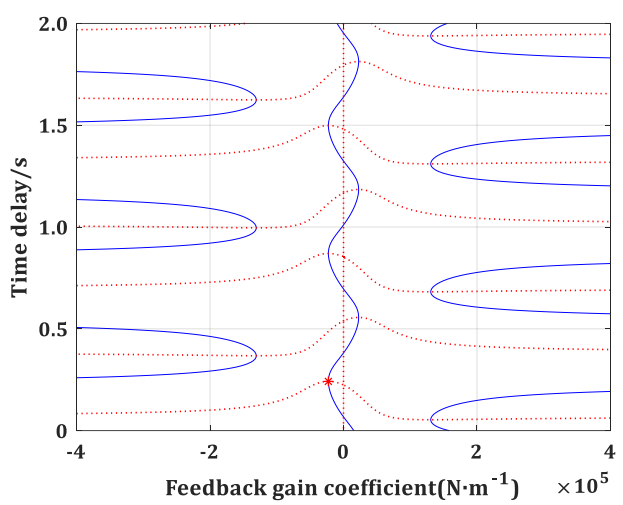

FIGURE 3: Extreme Point Distribution

TABLE 1: Simplified Vehicle Parameters

\begin{tabular}{|c|c|c|c|}
\hline $\begin{array}{c}\text { Vehicle } \\
\text { parameters }\end{array}$ & Value & $\begin{array}{c}\text { Vehicle } \\
\text { parameters }\end{array}$ & Value \\
\hline$m_{e} / \mathrm{kg}$ & 345 & $k_{2} /\left(N \cdot \mathrm{m}^{-1}\right)$ & 192000 \\
\hline$m_{c} / \mathrm{kg}$ & 40.5 & $c_{1} /\left(N \cdot s \cdot \mathrm{m}^{-1}\right)$ & 1500 \\
\hline$k_{1} /\left(N \cdot \mathrm{m}^{-1}\right)$ & 17000 & $c_{2} /\left(N \cdot s \cdot \mathrm{m}^{-1}\right)$ & 0 \\
\hline
\end{tabular}

\section{SIMULATION ANALYSIS}

\section{Frequency Domain Analysis}

The optimal time-delay feedback control parameters are introduced into equation (3), and the amplitudefrequency characteristic diagram based on the body displacement is obtained, as shown in FIGURE 4.

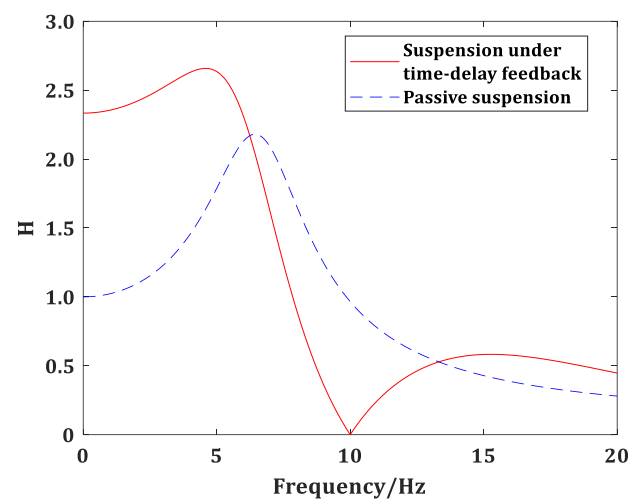

(a) Vehicle Body

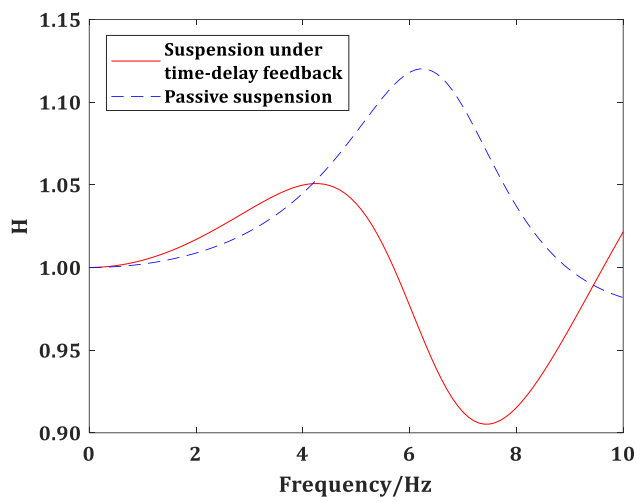

(b) Wheel

FIGURE 4: Amplitude Frequency Characteristic Diagram

It can be seen from FIGURE 4 (a) that under the time-delay feedback control, the body amplitude is greatly attenuated within $6.2 \mathrm{~Hz} \sim 13.4 \mathrm{~Hz}$, and the vibration is completely suppressed at $10 \mathrm{~Hz}$. It can be clearly seen from FIGURE 4 (b) that the time-delay feedback control can significantly improve the amplitude-frequency characteristics of the tire, especially in the low frequency range

\section{Time Domain Analysis}

Time-domain simulation of system vibration response was carried out in the MATLAB/Simulink module. $x_{d}=0.05 \times$ $\sin (10 t)$ with harmonic excitation was taken as the input of road vibration, and the optimal time-delay control parameters were substituted into the system. The simulation figure is shown in FIGURE 5.

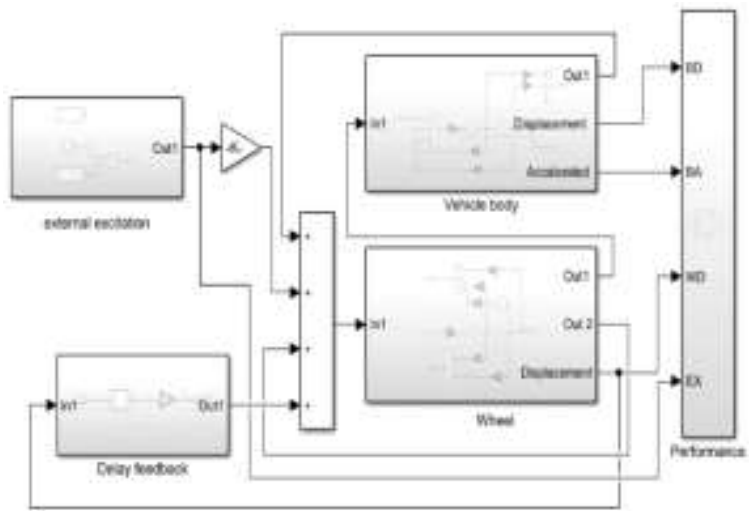

FIGURE 5: Simulation Model 


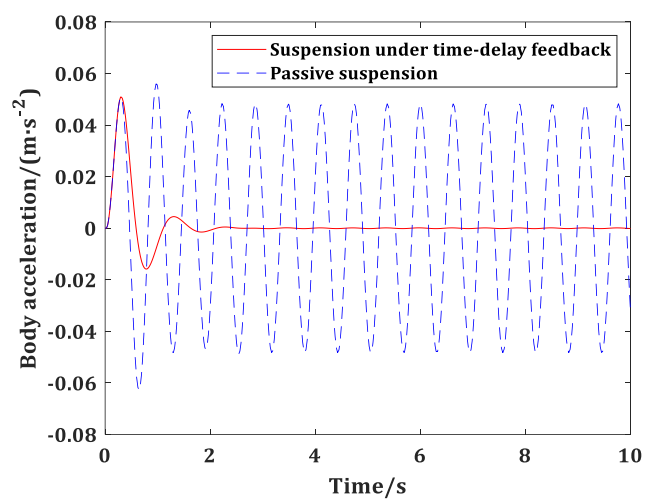

(a) Body Displacement Response

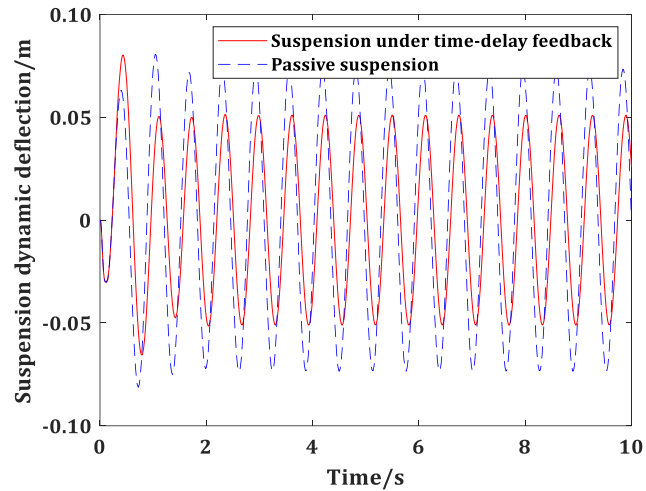

(c) Suspension Dynamic Deflection Response

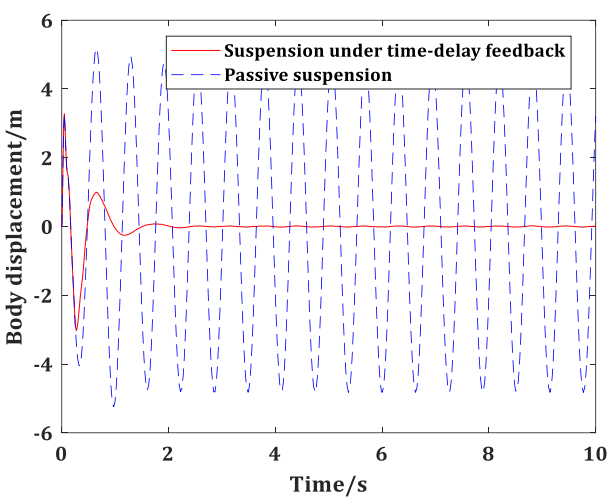

(b) Body Acceleration Response

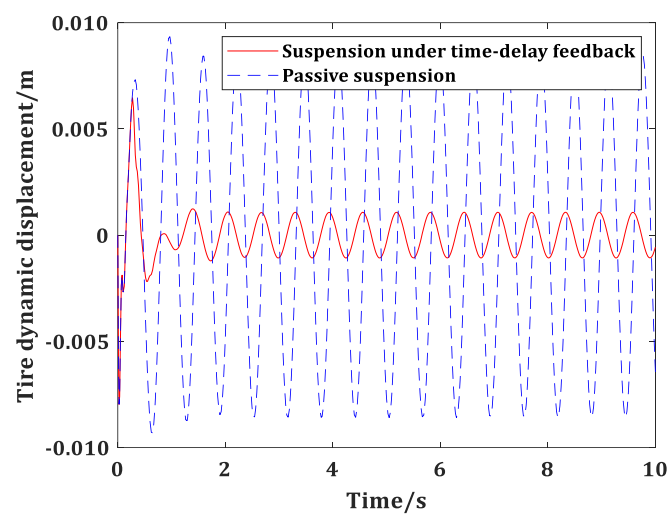

(d) Tire Dynamic Displacement Response

FIGURE 6: Simulation Comparison Under Simple Harmonic Excitation

It can be seen from FIGURE 6 that compared to passive suspension, when the road excitation frequency is close to the natural frequency of the vehicle body, the body displacement and body acceleration are significantly reduced, and the suspension dynamic deflection and tire dynamic displacement are also reduced to varying degrees. According to the root mean square (RMS) value of each vehicle performance in TABLE 2 , under the timedelay feedback control, the RMS of body displacement in FIGURE 4 (a) drops

TABLE 2: RMS Value of Vehicle Performance Indicators

\begin{tabular}{|l|l|c|c|}
\hline \multicolumn{1}{|c|}{ performance index } & \multicolumn{1}{|c|}{$\begin{array}{c}\text { passive } \\
\text { suspension }\end{array}$} & $\begin{array}{c}\text { Suspension under } \\
\text { time-delay } \\
\text { feedback }\end{array}$ & Reduction ratio \\
\hline RMS of body displacement $m$ & 0.0333 & 0.0082 & $75.39 \%$ \\
\hline RMS of body acceleration $m \cdot s^{-2}$ & 3.2682 & 0.4669 & $85.71 \%$ \\
\hline RMS of suspension dynamic deflection $m$ & 0.0513 & 0.0381 & $25.83 \%$ \\
\hline RMS of tire dynamic displacement $m$ & 0.0058 & 0.0012 & $79.10 \%$ \\
\hline
\end{tabular}

from 0.0333 to 0.0082 , and the reduction ratio is $85.71 \%$. The RMS of body acceleration in FIGURE 4 (b) drops from 3.2682 to 0.4669 , and the reduction ratio is $85.71 \%$. The RMS of suspension dynamic deflection in FIGURE 4 (c) drops from 0.0513 to 0.0381 , and the reduction ratio is 25.83\%. The RMS of dynamic displacement in FIGURE 4 (d) drops from 0.0058 to 0.0012 , and the reduction ratio is $79.10 \%$

From the above analysis, it can be concluded that the timedelay feedback control can effectively improve the damping performance of the suspension system and the grounding performance of the tires, and improve the ride comfort of the vehicle.

\section{CONCLUSIONS}

In this paper, time-delay state feedback is introduced into the $1 / 4$ vehicle model to conduct stability analysis and simulation of the time-delay control system, and the following conclusions are drawn:

(1) The time-delay feedback control can effectively reduce the acceleration of the vehicle body and improve the vibration damping performance of the vehicle suspension. At the same time, tire vibration has not deteriorated, improving the ride comfort of the vehicle.

(2) In the time-delay feedback control, if the control parameters are not selected properly, the system may be unstable and the vibration reduction effect may become worse. 


\section{REFERENCES}

[1] Liu JJ, Sun YX, Li S. Time delay feedback control and parameter optimization of automotive suspension system. Journal of Mechanical \& Electrical Engineering, 2020, 37(1): 54-58.

[2] Olgac N, Holm-Hansen BT. A novel active vibration absorption technique: delayed resonator. Journal of Sound and Vibration, 1994, 176(1):93-104.

[3] Olgac N, Holm-Hansen BT. Design considerations for delayed-resonator vibration absorbers. Journal of Engineering Mechanics, 1995. 121:80-89.

[4] Jalili, Olgac N. A quasi online tuning structure for optimum delayed feedback vibration absorber. Proceedings of American Control Conference. San Diego, California. 1999. 1550-1554.
[5] Zhao YY, Xu J. Vibration reduction mechanism of time-delay nonlinear dynamic vibration absorber. Chinese Journal of Theoretical and Applied Mechani. 2008, 40(1): 98-106.

[6] Li S, Sun YX. Application of LQR control and timedelay feedback control in vehicle vibration reduction. Software. 2020, 41(2): 12-17.

[7] Zhao YY, Xu J. Effects of delayed feedback control on nonlinear vibration absorber system. J Sound Vid. 2007, 38: $212-230$

[8] Liu L. Time-delay vibration reduction mechanism and its application in vehicles. Master's Thesis. China, 2012. 